(C) This is a U.S. government work and not under copyright protection in the U.S.; foreign copyright protection may apply, 2021

DOI: $10.1007 / \mathrm{s} 13592-021-00877-3$

\title{
Nutrigenetic comparison of two Varroa -resistant honey bee stocks fed pollen and spirulina microalgae
}

\author{
Vincent A Ricigliano (iD, Kate E Ihle, Steven T Williams \\ Honey Bee Breeding, Genetics and Physiology Research, USDA-ARS, Baton Rouge, LA 70820, USA
}

Received 12 March 2021 - Revised 13 May 2021 - Accepted 13 June 2021

\begin{abstract}
We tested the influence of genetic variation on responses to natural and artificial diets in Varroa resistant Pol-line and Russian honey bee stocks. Newly emerged workers from six colonies per stock were fed pollen, spirulina (blue-green microalgae), and sucrose-only diets in 144 total cages. Diet type had a strong effect on sugar intake, body weight, fat body lipid content, and vitellogenin ( $v g$ ) expression. Spirulina consumption was approximately half that of pollen, but led to higher head weights, equivalent thorax weights and $v g$ levels, and marginally reduced fat body lipids. Bee stock and colony had a significant impact on nutritional response. Despite equivalent diet intakes, Pol-line bees accumulated higher lipid levels and consumed less sugar overall than Russian bees. Furthermore, pollen-fed bees sourced from Pol-line colonies had significantly higher vg levels. These differences in nutrient and energy allocation may reflect life history-related physiological tradeoffs. Our results suggest that genotype-dependent nutritional responses are present in honey bees, with promising implications for breeding efforts and tailored approaches to diet and health in a changing global climate.
\end{abstract}

Apis mellifera , / nutritional genetics, / breeding, / lipids, / vitellogenin

\section{INTRODUCTION}

Nutritional health is determined by interactions between environmental variables of supply, bioavailability, and consumption of diet components as well as the genetically controlled variables of digestion, absorption, transformation, and storage. Technical advances have accelerated the study of nutrition at the molecular level and inherited traits at the DNA level. This research has led to investigation of the roles of nutrients in gene and protein expression known as nutrigenomics, and concepts related to genetic variation and dietary response, known as nutrigenetics (e.g., different individual responses to the same diet leading to different levels of physiological biomarkers due to genetic variation, Simopoulos 2010). Nutrigenomics/

Corresponding author: V. A. Ricigliano,

Vincent.Ricigliano@USDA.gov

Handling Editor: Yves Le Conte nutrigenetics has been successfully applied to increase growth rates and productivity in livestock (Scholtz et al. 2013; Benitez et al. 2017), and could provide a framework for breeding improved nutrient efficiency as well as identification of genotypedependent supplements that promote health (Trujillo et al. 2006; Ferguson 2009; Tallentire et al. 2016). The honey bee is a promising model for such studies since its nutritional requirements are well characterized and a variety of genomic, transcriptomic, and proteomic resources are available (Alaux et al. 2011; Chakrabarti et al. 2019; Rutter et al. 2019; Ricigliano et al. 2020).

Floral nectar serves as an energy source while pollen provides all amino acids, lipids, and micronutrients necessary for honey bee development and reproduction (Brodschneider and Crailsheim 2010). The protein content of pollen and its relative abundances of essential amino acids are central to brood production and colony growth (De Groot 1953; Crailsheim 1990). Pollen 
also contains essential lipids (Ischebeck 2016) that are incorporated into abdominal stores associated with reproductive potential (Wegener et al. 2018; Martin et al. 2019). To supplement colony nutrition during periods of reduced natural forage, managed honey bee colonies are often fed artificial pollen substitute diets (Nabors 2000). This management practice has become more prevalent as commercial beekeeping has grown in scale, and landscapes increase in agricultural intensity, which reduces floral diversity and consequent nutritional value (Naug 2009). Artificial bee diets typically incorporate proteins derived from plant (e.g., soy, corn, or wheat), animal (e.g., milk or egg), and microbial sources (e.g., brewer's yeast). Additionally, microalgae have recently emerged as a promising pollen substitute due to their high macronutrient content and bioavailability (Ricigliano 2020).

A variety of bee responses to pollen and artificial diets have been described, but comparatively fewer studies have focused on molecular impacts of nutrition. For example, pollen and microalgae diets broadly upregulate metabolic processes associated with amino acids, lipids, and carbohydrates (Azzouz-Olden et al. 2018; DeGrandiHoffman et al. 2018; Ricigliano et al. 2020). These diets particularly upregulate protein expression associated with longevity and stress response, including vitellogenin, a multifunctional lipoprotein involved in nutritional storage, brood production, stress response, and overwintering (Amdam et al. 2003; Amdam et al. 2005; Smart et al. 2016). Vitellogenin titers are positively linked to abdominal lipid content and to the timing and quantity of diet consumption (Wegener et al. 2018). Therefore, vitellogenin expression at the mRNA and protein levels can be considered a biomarker of honey bee nutritional status (Alaux et al. 2011;).

Despite a sizable body of research pertaining to honey bee nutrition, relatively little is known about the effects of genetic variation on nutritional response. A number of honey bee stocks (isolated populations bred for specific traits) are produced by queen breeders and distributed throughout the US (Delaney et al. 2009; Saelao et al. 2020). Some of these stocks have been developed for specialized traits (e.g; mite resistance) or are the product of research-based breeding programs (Page and Fondrk 1995; Spivak and Reuter 2001; Rinderer et al. 1997; Danka et al. 2016). Understanding honey bee genotype-dependent responses to nutrition could enable marker-assisted breeding for improved health outcomes or the development of tailored feed supplements. The effects of diet have been studied in other livestock animals with reference to effective breeding and their influence on production traits and health (Nowacka-Woszuk 2020). The objective of this study was to test the effects of honey bee genetic variation on responses to natural and artificial diets. Two Varroa -resistant honey bee stocks, Pol-line and Russian, were selected for this study based on their closed breeding populations and genomic distinction. Using cage bioassays, nutritional response variables were measured in young worker bees fed bee-collected pollen and an artificial microalgae (spirulina)-based diet.

\section{MATERIALS AND METHODS}

\subsection{Honey bees and experimental design}

Experiments were conducted at the USDAARS honey bee lab in Baton Rouge, LA, USA. Six healthy colonies each of Pol-line and Russian honey bee stocks were selected. Newly emerged workers ( $<24 \mathrm{~h}$ old) were obtained by incubating sealed brood combs overnight at $35^{\circ} \mathrm{C}$ and $50 \%$ $\mathrm{RH}$. Bees from each colony were collected into separate containers and then assigned to diet treatment cages ( 50 bees per cage and 144 total cages, see Figure 1 for experimental design schematic). Bees were collected from each cage at day 7 for nutritional response measures. Dead bees were counted and removed from the cages daily. Mortality was less than $1 \%$ overall during the 7-day trial, so mortality was negligible.

\subsection{Diet preparation and feeding assay}

All cages were provided ad libitum access to drip feeders containing $50 \%(\mathrm{w} / \mathrm{v})$ sucrose solution. Cages were fed ad libitum diets of beecollected pollen, spirulina microalgae, or sucrose only. Pollen and spirulina diets were mixed into a paste with $50 \%$ sucrose $(\mathrm{w} / \mathrm{v})$ syrup containing 


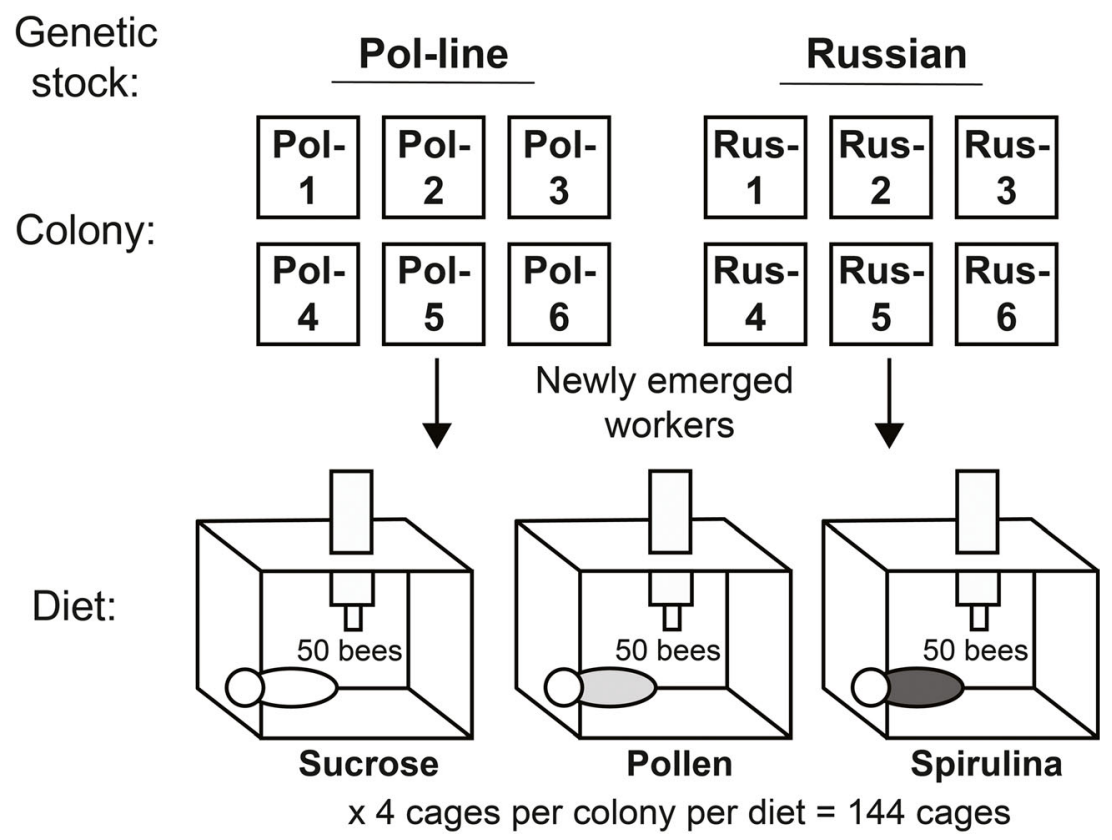

Figure 1. Schematic overview of the experimental design used in this study. Newly emerged workers from six colonies each of Pol-line and Russian honey bee stocks were used in cage feeding bioassays to test the effects of genetic variation on nutritional response.

$5 \%$ glycerol (v/v; to improve pliability) and loaded into modified 1.7-mL microcentrifuge tubes (see Figure 1 for cage setup schematic), then stored at $-20{ }^{\circ} \mathrm{C}$ before use. For the pollen diet, mixed corbicular pollen pellets were collected using entrance-mounted pollen traps in a USDAARS apiary in Baton Rouge and immediately frozen until ready for use (Mogren et al. 2018). Spirulina diet contained organic, dried spirulina (Arthrospira platensis) powder (Microingredients, California, USA). Approximately $1.5 \mathrm{~g}$ of diet paste was provided to each cage. The amount of diet consumed by each cage was recorded on day 3 , then the diet was refreshed with $\sim 1.5 \mathrm{~g}$ of new diet paste and consumption was measured again at day 7 . As a control, diet samples were placed in cages without bees and weight loss was measured to determine the evaporation rate for each diet type. Diet consumption in each cage was adjusted for daily moisture loss and recalculated to give the total diet consumed over the 7 day period per bee. Sugar consumption was recorded and expressed as the total amount consumed over 7 days per bee for each cage.

\subsection{Nutritional physiology measures}

Bees were dissected into head, thorax (excluding legs and wings), and abdomens with guts removed. Then, dissected parts were collected into pools of 8 . Average head and thorax weights per bee for each cage were determined by drying to a constant weight $\left(60^{\circ} \mathrm{C}\right.$ for $\left.48 \mathrm{~h}\right)$ and recording to the nearest $0.1 \mathrm{mg}$. Average abdomen lipid content per bee for each cage was determined by homogenizing pooled abdomens in $800 \mu \mathrm{l}$ of TE buffer, centrifugation, then extracting $300 \mu$ l of supernatant and combining it with $300 \mu$ of $2: 1$ of chloroform:methanol. Abdomen samples from pollen- and spirulina-fed bees required a further 1:1 dilution with chloroform. Samples were centrifuged and $250 \mu \mathrm{l}$ of the chloroform layer was added to glass tubes and incubated at $95^{\circ} \mathrm{C}$ to evaporate the chloroform. Next, $300 \mu$ l of concentrated sulfuric acid (98\%) was added and the sample was incubated for $20 \mathrm{~min}$. Then, 100ul of sample was transferred in technical duplicates to a microplate. The remaining steps were carried out on according to Cheng et al. 2011. Optical 
reads of the lipid samples were compared with those measured for a canola oil standard ran on each plate. A standard curve was generated based on the average of standards ran on all plates and was used to calculate the lipid content of the samples. The $R^{2}$ value for the lipid standard curve was 0.99 (Online resource 1 Figure S1).

\subsection{Vitellogenin (vg) and vg-like-A expression}

For gene expression analyses, flash frozen pools of 8 bee abdomens per cage were homogenized in $700 \mu \mathrm{l}$ of Maxwell@ simplyRNA homogenization solution (Promega), centrifuged, and $400 \mu \mathrm{l}$ of the supernatant was transferred to Maxwell@ RSC-48 cartridges (Promega). RNA extractions proceeded according to the Maxwell® RSC simplyRNA tissue kit protocol (Promega).

cDNA synthesis was carried out using $100 \mathrm{ng}$ of DNAase (New England Biolabs)treated RNA and QuantiTect Reverse Transcription Kits (Qiagen) according to the manufactures protocol. Quantitative PCR (qPCR) was carried out in triplicate using previously published primer pairs and cycling conditions to quantify expression levels of vitellogenin $(v g)$ and its homologue vg-like- $A$ (Salmela et al. 2016) using $\beta$-actin and RP-49 for normalization (Lourenço et al. 2008)

\subsection{Statistical analyses}

The effects of bee stock (Pol-line, Russian), colony (Pol-line 1-6, Russian 1-6), and diet type (sucrose only, pollen, spirulina) were evaluated using mixed-model ANOVA with post hoc contrasts of least squares mean differences as required (Figures 2, 3, 4 and 5). Dependent variables were evaluated for normality using fit statistics and probability plots. Variables with deviations from normality were re-evaluated after log transformation.

Secondary analyses were performed within groups of either pollen- or spirulina-fed bees to test the effects of bee stock and colony using nested ANOVA (Tables I, II, III and IV). Analyses were conducted in JMP v11 and Prism v7.

\section{RESULTS}

\subsection{Diet and sugar consumption}

Diet consumption was influenced by diet type $\left(F_{1,92}=218.5, P<0.0001\right)$ but not by stock or stock*diet interaction. Average consumption was significantly higher for the pollen diet (Pol-line: $41.60 \mathrm{mg} / \mathrm{bee}$; Russian: $43.83 \mathrm{mg} / \mathrm{bee}$ ) relative to the spirulina diet (Pol-line: $22.53 \mathrm{mg} /$ bee; Russian: $24.14 \mathrm{mg} / \mathrm{bee}$ ) (Figure 2).

Among pollen-fed bees, diet consumption was influenced by colony $\left(F_{10,36}=218.5, P=\right.$ 0.0024 ) (Table I) but not by stock. Among spirulina-fed bees, stock and colony did not significantly impact diet consumption (Table I).

Sugar consumption was higher in Russian bees $\left(\mathrm{F}_{1,142}=16.3, P=0.0024\right)$ and was influenced by $\operatorname{diet}\left(F_{2,141}=200.9, P<0.0001\right)$ but not by stock*diet interaction (Figure 2 ). Ranked by diet type, sugar consumption was as follows: sucrose $(103.09 \mathrm{mg} / \mathrm{bee})>$ spirulina $(82.81 \mathrm{mg} / \mathrm{bee})>$ pollen (63.54 mg/bee) (Figure 2).

Among pollen-fed bees, sugar consumption was higher in Russian stock $\left(F_{1,46}=34.9, P<\right.$ $0.0001)$ and was influenced by colony $\left(F_{10,36}=\right.$ $4.3, P=0.0005$ ) (Table I). Among spirulina-fed bees, sugar consumption was also higher in Russian stock $\left(F_{1,46}=23.3, P<0.0001\right)$, but was not influenced by colony (Table I).

\subsection{Head and thorax weight}

Head weight was influenced by diet type $\left(F_{2}\right.$, $\left.{ }_{138}=99.3, P<0.0001\right)$ but not by stock or stock*diet interaction, although newly emerged Pol-line bees had higher head weights then Russian bees (Figure 3, Online resource 1 Figure S2). Average head weight was highest in spirulina-fed bees (Pol-line: $3.03 \mathrm{mg} /$ bee DW; Russian: 2.91 $\mathrm{mg} /$ bee DW), followed by pollen-fed bees (Polline: $2.88 \mathrm{mg} /$ bee DW; Russian: $2.73 \mathrm{mg} / \mathrm{bee}$ DW), followed by sucrose-fed bees (Pol-line: $2.37 \mathrm{mg} /$ bee DW; Russian: $2.18 \mathrm{mg} /$ bee DW) (Figure 3).

Among pollen-fed bees, head weight was higher in Pol-line stock $\left(F_{1,46}=7.8, P=\right.$ $0.0084)$ and was influenced by colony $\left(F_{10,36}\right.$ $=2.9, P=0.0085$ ) (Table II). Among spirulina- 

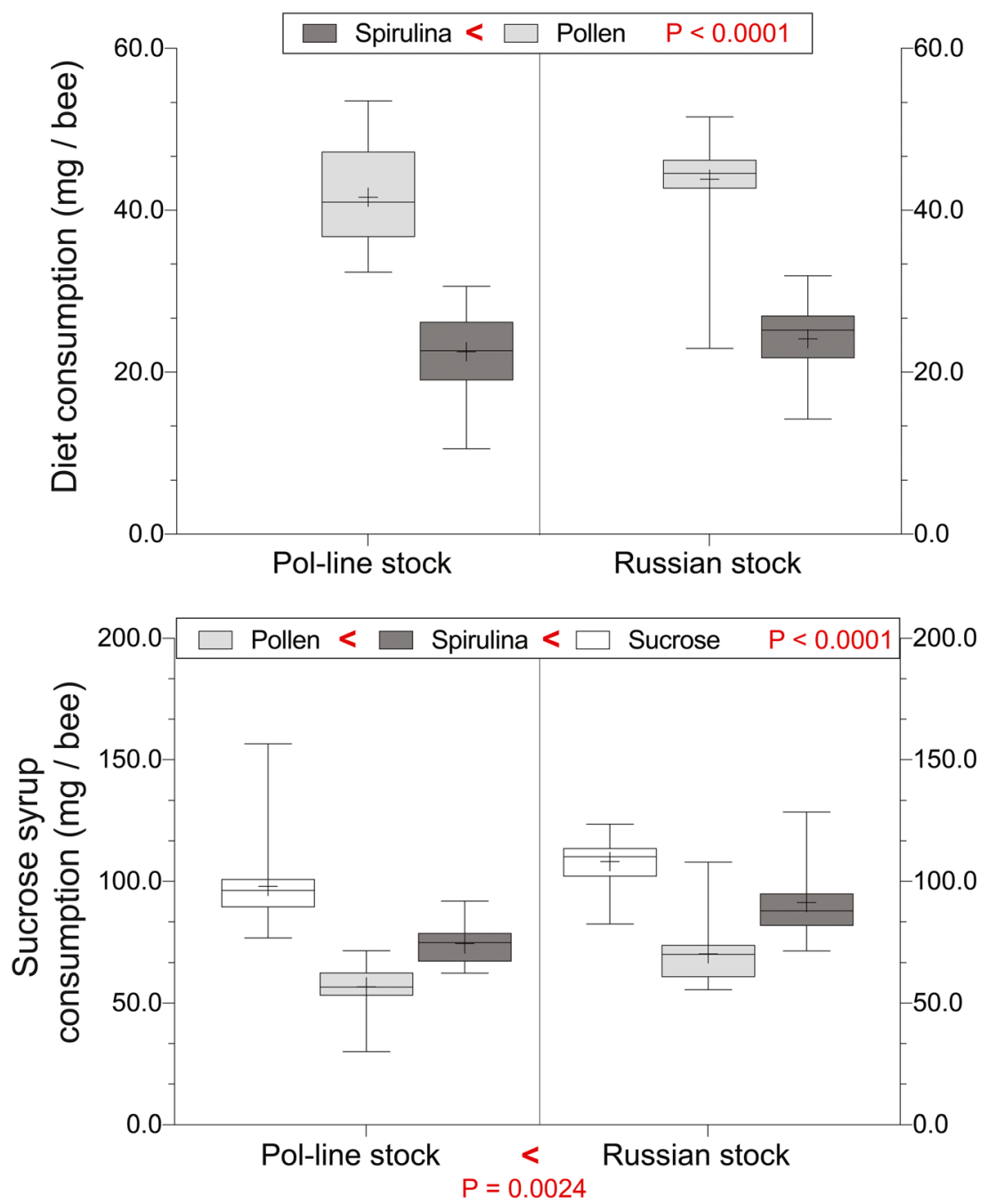

Figure 2. Ad libitum diet and sugar consumption by the two honey bee stocks after 7 days. Plot whiskers show minimum and maximum values with the lower and upper edge of each box denoting the 25th to 75th percentiles, median as a horizontal bar, and "+" as the mean. Main effects determined by mixed model ANOVA are indicated in red.

fed bees, head weight was influenced by colony $\left(F_{10,35}=2.5, P=0.0208\right)$ but not by stock (Table II). There was a significant, positive correlation between head weight and diet consumption in Pol-line but not Russian stock (Online resource 1 Figure S3).

Thorax weight was influenced by diet type ( $F$ $2,138=71.3, P<0.0001)$, but not by stock or by stock*diet interaction (Figure 3 ). Newly emerged Pol-line bees had higher thorax weights than Russian bees (Online resource 1 Figure S4). Average thorax weight was not significantly different between bees fed pollen (Pol-line: $9.73 \mathrm{mg} /$ bee DW; Russian: $9.23 \mathrm{mg} / \mathrm{bee} \mathrm{DW}$ ) and spirulina (Polline: $9.85 \mathrm{mg} /$ bee DW; Russian: $9.33 \mathrm{mg} / \mathrm{bee}$ DW) but was lowest in sucrose-fed bees (Pol-line: $8.93 \mathrm{mg} /$ bee DW; Russian: $8.09 \mathrm{mg} /$ bee DW).

Among pollen-fed bees, thorax weight was higher in Pol-line stock ( $\mathrm{F}_{1,46}=24.1, P=$ $0.0018)$ and was influenced by colony $\left(F_{10}\right.$, ${ }_{36}=2.5, P=0.0207$ ) (Table II). A significant, positive correlation was determined 

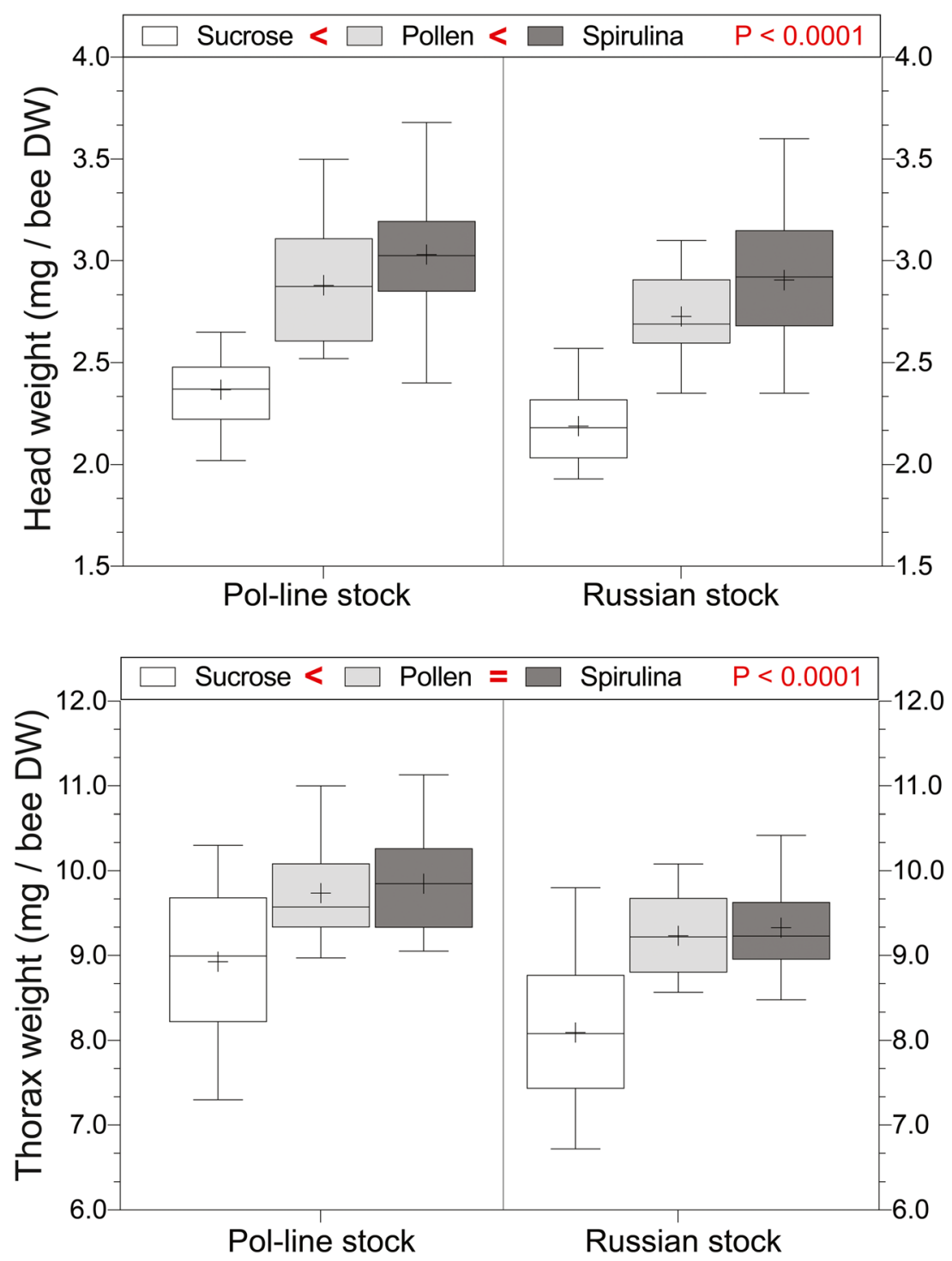

Figure 3. Head and thorax weights of the two honey bee stocks after 7 days of diet consumption. Plot whiskers show minimum and maximum values with the lower and upper edge of each box denoting the 25th to 75th percentiles, median as a horizontal bar, and " + " as the mean. Main effects determined by mixed model ANOVA are indicated in red.

between thorax weight and pollen consumption in Russian but not Pol-line stock (Online resource 1 Figure S5). Among spirulina-fed bees, thorax weight was higher in Pol-line stock $\left(F_{1,46}=11.4, P<0.0001\right)$ and was influenced by colony $\left(F_{10}, 36=3.5, P=\right.$ 0.0030) (Table II).

\subsection{Abdominal lipid content}

Abdomen lipid content was higher in Pol-line bees $\left(F_{1,139}=8.7, P=0.0144\right)$ and was influenced by $\operatorname{diet}\left(F_{2,138}=44.8, P=0.0144\right)$ but not stock*diet interaction (Figure 4 ). Ranked by diet, average lipid content was as follows: pollen-fed bees $(591.48 \mu \mathrm{g} / \mathrm{bee})>$ spirulina-fed bees $(511.79$ $\mu \mathrm{g} / \mathrm{bee})>$ sucrose-fed bees $(310.21 \mu \mathrm{g} / \mathrm{bee})$. 


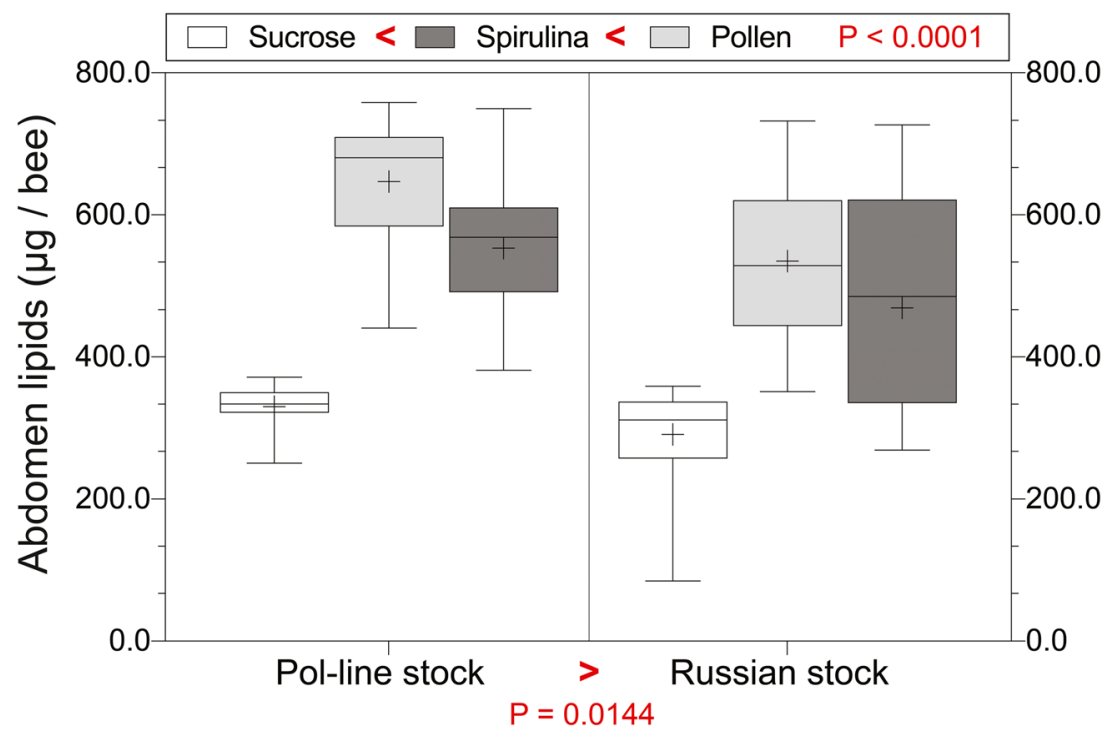

Figure 4. Abdominal lipid accumulation in the two honey bee stocks after 7 days of diet consumption. Plot whiskers show minimum and maximum values with the lower and upper edge of each box denoting the 25th to 75th percentiles, median as a horizontal bar, and "+" as the mean. Main effects determined by mixed model ANOVA are indicated in red.

Among pollen-fed bees, lipid content was higher in Pol-line stock $\left(F_{1,46}=25.5, P<\right.$ $0.0001)$ and was influenced by colony $\left(F_{10,36}\right.$ $=4.3, P=0.0006$ ) (Table III). Among spirulinafed bees, lipid content was also higher in Pol-line stock $\left(F_{1,46}=12.1, P=0.0014\right)$ and influenced by colony $\left(F_{10,36}=6.3, P<0.0001\right)$ (Table III).

\subsection{Vitellogenin (vg) and vg-like-A expression}

Vitellogenin ( $v g$ ) expression was influenced by diet $\left(F_{2,139}=179.9, P<0.0001\right)$ but not by stock or stock*diet interaction (Figure 5). Vg expression levels were not significantly different between bees fed pollen and spirulina, but were lowest in sucrose-fed bees.

Among pollen-fed bees, vg expression was higher in Pol-line stock $\left(F_{1,46}=7.6, P<\right.$ $0.0095)$ and was influenced by colony $\left(F_{10,35}\right.$ =7.9, $P<0.0001$ ) (Table IV). A significant positive correlation was determined between diet consumption and $v g$ expression in Pol-line stock, and trended toward significance in Russian stock (Online resource 1 Figure S6). Among spirulina- fed bees, $v g$ levels were influenced by colony ( $F$ $10,35=3.0, P=0.0071)$ but not stock (Table IV).

$V g$-like-A expression was influenced by diet $\left(F_{2,140}=68.9, P<0.0001\right)$ but not by stock or stock*diet interaction (Figure 5). Vg-like-A levels in bees fed pollen and spirulina were not different from each other, but were significantly higher than sucrose-fed bees (Figure 5).

Among pollen-fed bees, vg-like- $A$ expression was higher in Russian stock ( $\mathrm{F}_{1,46}=4.6, P=$ $0.0398)$ and was influenced by colony $\left(F_{10,35}=\right.$ 7.4, $P<0.0001$ ) (Table IV). Among spirulina-fed bees, $v g$-like- $A$ expression was not influenced by stock or colony (Table IV).

\section{DISCUSSION}

This study compared nutritional responses of two honey bee stocks fed pollen and spirulina, a microalgae-based artificial feed. The Pol-line stock was developed by USDA-ARS via introgression of the Varroa Sensitive Hygiene trait (Harbo and Harris 2005) into Italian bees managed by several US commercial beekeepers (Danka et al. 2016). The Russian honey bee 


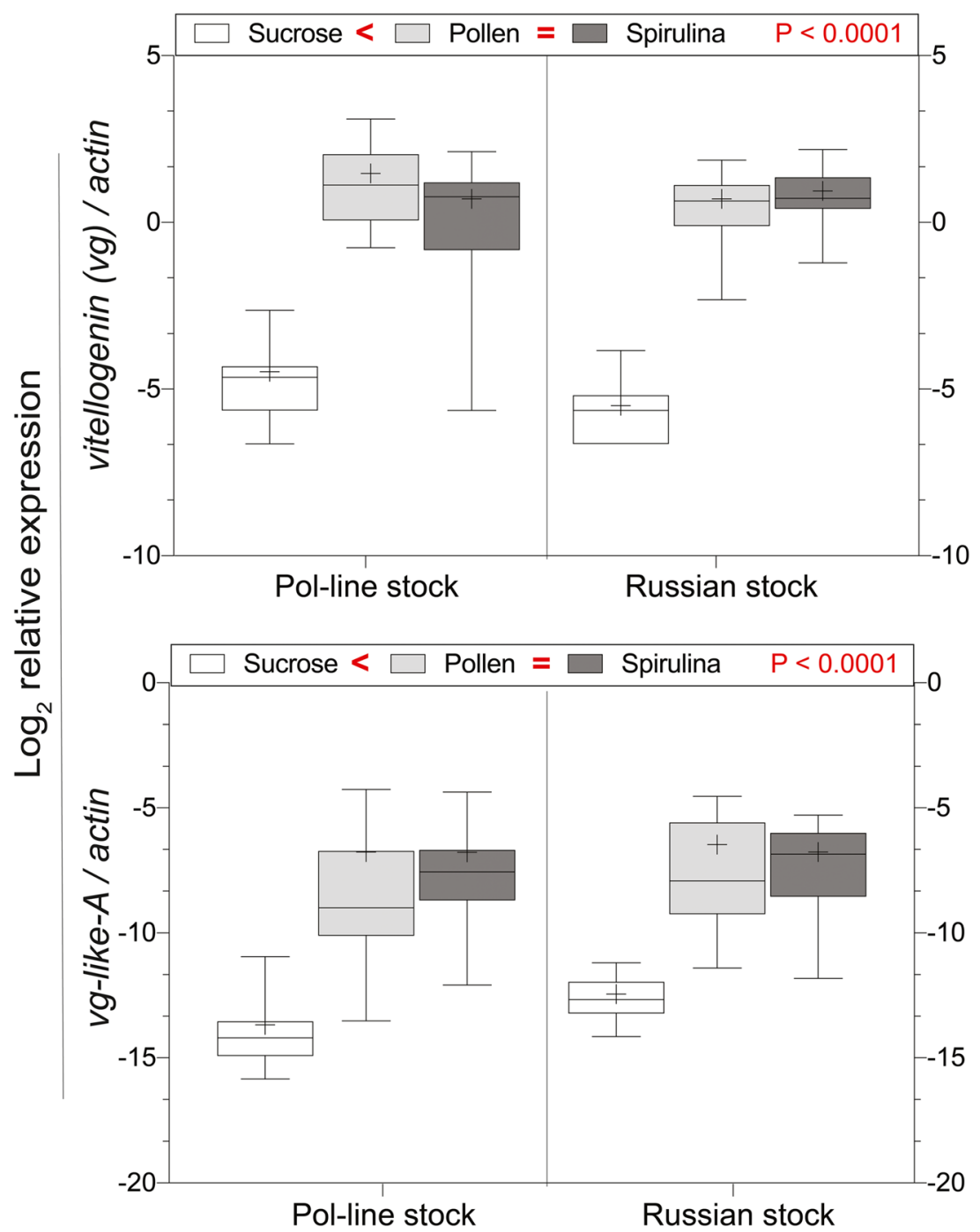

Figure 5. Relative mRNA expression levels of vitellogenin $(v g)$ and its homologue $v g$-like- $a$ in the two honey bee stocks after 7 days of diet consumption. Plot whiskers show minimum and maximum values with the lower and upper edge of each box denoting the 25 th to 75 th percentiles, median as a horizontal bar, and " + " as the mean. Main effects determined by mixed model ANOVA are indicated in red.

breeding program was established by USDAARS via importation of queens from eastern Russia for the purpose of genetic improvement of Varroa resistance (Rinderer et al. 1997). Polline and Russian stocks were chosen for this study based on closed breeding programs and recent evidence confirming their genomic distinction (Saelao et al. 2020). We identified genotypedependent nutritional responses, and these findings warrant further investigation of additional honey bee populations at individual and colony levels. Genetic and environmental factors influence nutrient and energy allocation for specific physiological functions at different life stages of homometabolous insects (Nestel et al. 2016). A range of metabolic regulatory mechanisms can affect all developmental stages, and ultimately fitness (Kaspi et al. 2002). Organization of the honey bee colony is often referred to as a superorganism, in which workers perform all functions 
Table I. Average diet and sugar consumption by newly emerged workers from Pol-line and Russian colonies after 7 days

\begin{tabular}{|c|c|c|c|c|c|c|c|c|}
\hline \multirow{3}{*}{$\begin{array}{l}\text { Colony } \\
\text { Pol-1 }\end{array}$} & \multicolumn{3}{|c|}{$\underline{\text { Feed consumption }(\mathrm{mg} / \mathrm{bee} \pm \mathrm{SE})}$} & & \multicolumn{4}{|c|}{$\underline{\text { Sugar consumption }(\mathrm{mg} / \mathrm{bee} \pm \mathrm{SE})}$} \\
\hline & \multicolumn{2}{|l|}{ Pollen-fed } & \multicolumn{2}{|l|}{ Spirulina-fed } & \multicolumn{2}{|l|}{ Pollen-fed } & \multicolumn{2}{|l|}{ Spirulina-fed } \\
\hline & $44.54 \pm 3.37$ & $a b c$ & $26.27 \pm 0.98$ & a & $56.20 \pm 1.46$ & $\mathrm{bc}$ & $73.58 \pm 2.06$ & $a b$ \\
\hline Pol-2 & $48.17 \pm 2.25$ & $\mathrm{a}$ & $25.75 \pm 2.06$ & a & $52.88 \pm 2.76$ & $\mathrm{c}$ & $71.93 \pm 3.54$ & $\mathrm{~b}$ \\
\hline Pol-3 & $35.14 \pm 1.05$ & $\mathrm{c}$ & $19.48 \pm 1.66$ & a & $59.84 \pm 3.36$ & bc & $70.59 \pm 2.57$ & $\mathrm{~b}$ \\
\hline Pol-4 & $44.40 \pm 2.11$ & $a b c$ & $23.80 \pm 2.94$ & $\mathrm{a}$ & $52.46 \pm 8.11$ & $\mathrm{c}$ & $80.47 \pm 4.49$ & $\mathrm{ab}$ \\
\hline Pol-5 & $36.05 \pm 1.61$ & $\mathrm{bc}$ & $19.35 \pm 1.35$ & a & $60.53 \pm 5.31$ & $\mathrm{bc}$ & $74.30 \pm 6.64$ & $\mathrm{ab}$ \\
\hline Pol-6 & $41.27 \pm 1.55$ & $a b c$ & $23.07 \pm 1.54$ & a & $58.63 \pm 3.29$ & $\mathrm{bc}$ & $75.48 \pm 4.17$ & $a b$ \\
\hline Rus-1 & $47.08 \pm 1.55$ & $a b$ & $22.08 \pm 4.75$ & a & $71.93 \pm 0.83$ & $a b c$ & $94.52 \pm 9.95$ & $a b$ \\
\hline Rus-2 & $45.35 \pm 2.33$ & $a b c$ & $19.36 \pm 2.18$ & $\mathrm{a}$ & $60.94 \pm 3.15$ & $\mathrm{bc}$ & $86.55 \pm 9.43$ & $a b$ \\
\hline Rus-3 & $45.97 \pm 0.57$ & $a b c$ & $24.62 \pm 1.23$ & $\mathrm{a}$ & $60.62 \pm 1.37$ & $\mathrm{bc}$ & $86.47 \pm 2.78$ & $a b$ \\
\hline Rus-4 & $42.80 \pm 0.53$ & $a b c$ & $27.01 \pm 2.40$ & $\mathrm{a}$ & $64.84 \pm 1.78$ & $\mathrm{bc}$ & $83.69 \pm 2.51$ & $a b$ \\
\hline Rus-5 & $37.88 \pm 5.19$ & $a b c$ & $26.90 \pm 1.14$ & $\mathrm{a}$ & $73.40 \pm 2.17$ & $\mathrm{ab}$ & $94.15 \pm 7.18$ & $\mathrm{ab}$ \\
\hline Rus-6 & $43.95 \pm 0.91$ & $a b c$ & $20.78 \pm 2.18$ & $\mathrm{a}$ & $90.20 \pm 6.56$ & $\mathrm{a}$ & $102.1 \pm 8.93$ & $\mathrm{a}$ \\
\hline
\end{tabular}

Values within a column which have no letters in common are significantly different at $\alpha=0.05$

related to colony maintenance as they transition through different life history stages (Hölldobler and Wilson 2009). The superorganismal nature of a bee colony is particularly apparent in the context of nutrition. Young adult workers (nurse bees, $<14$ days old) play a central role since this age cohort of individuals assimilates, stores, and redistributes pollen-derived nutrition (Crailsheim et al. 1992). As such, we focused on nutritional responses of newly emerged workers after 7 days,

Table II. Average head and thorax weights of newly emerged workers from Pol-line and Russian colonies after 7 days of pollen and spirulina consumption

\begin{tabular}{|c|c|c|c|c|c|c|c|c|}
\hline \multirow{3}{*}{$\begin{array}{l}\text { Colony } \\
\text { Pol-1 }\end{array}$} & \multicolumn{3}{|c|}{$\underline{\text { Head weight }(\mathrm{mg} / \mathrm{bee} \mathrm{DW} \pm \mathrm{SE})}$} & & \multicolumn{4}{|c|}{$\underline{\text { Thorax weight }(\mathrm{mg} / \mathrm{bee} \mathrm{DW} \pm \mathrm{SE})}$} \\
\hline & \multicolumn{2}{|l|}{ Pollen-fed } & \multicolumn{2}{|l|}{ Spirulina-fed } & \multicolumn{2}{|l|}{ Pollen-fed } & \multicolumn{2}{|l|}{ Spirulina-fed } \\
\hline & $2.78 \pm 0.15$ & $\mathrm{ab}$ & $3.05 \pm 0.07$ & a & $9.18 \pm 0.05$ & $\mathrm{bc}$ & $9.24 \pm 0.11$ & $\mathrm{bc}$ \\
\hline Pol-2 & $2.90 \pm 0.13$ & $\mathrm{ab}$ & $2.95 \pm 0.08$ & a & $9.66 \pm 0.26$ & $a b c$ & $9.42 \pm 0.14$ & $a b c$ \\
\hline Pol-3 & $2.65 \pm 0.06$ & $a b$ & $2.88 \pm 0.17$ & a & $9.82 \pm 0.12$ & $a b c$ & $9.49 \pm 0.20$ & $a b c$ \\
\hline Pol-4 & $3.18 \pm 0.15$ & a & $2.98 \pm 0.20$ & a & $10.2 \pm 0.29$ & $\mathrm{ab}$ & $9.75 \pm 0.15$ & $a b c$ \\
\hline Pol-5 & $2.73 \pm 0.08$ & $a b$ & $2.98 \pm 0.08$ & a & $9.85 \pm 0.19$ & $\mathrm{abc}$ & $10.1 \pm 0.36$ & $\mathrm{ab}$ \\
\hline Pol-6 & $3.09 \pm 0.09$ & a & $3.35 \pm 0.18$ & a & $10.4 \pm 0.26$ & a & $10.4 \pm 0.21$ & a \\
\hline Rus-1 & $2.98 \pm 0.08$ & $a b$ & $3.31 \pm 0.14$ & $\mathrm{a}$ & $9.47 \pm 0.15$ & $a b c$ & $9.60 \pm 0.23$ & $a b c$ \\
\hline Rus-2 & $2.68 \pm 0.11$ & $a b$ & $2.67 \pm 0.18$ & $\mathrm{a}$ & $9.04 \pm 0.27$ & $\mathrm{c}$ & $8.98 \pm 0.26$ & $\mathrm{c}$ \\
\hline Rus-3 & $2.72 \pm 0.07$ & $a b$ & $3.08 \pm 0.16$ & $\mathrm{a}$ & $9.33 \pm 0.31$ & $a b c$ & $9.24 \pm 0.14$ & $\mathrm{bc}$ \\
\hline Rus-4 & $2.64 \pm 0.05$ & $a b$ & $2.69 \pm 0.07$ & $\mathrm{a}$ & $9.06 \pm 0.24$ & $\mathrm{c}$ & $9.13 \pm 0.08$ & $\mathrm{bc}$ \\
\hline Rus-5 & $2.76 \pm 0.06$ & $a b$ & $2.93 \pm 0.09$ & $\mathrm{a}$ & $9.55 \pm 0.15$ & $a b c$ & $9.87 \pm 0.31$ & $a b c$ \\
\hline Rus-6 & $2.52 \pm 0.09$ & $\mathrm{~b}$ & $2.70 \pm 0.13$ & $\mathrm{a}$ & $8.87 \pm 0.18$ & $\mathrm{c}$ & $9.02 \pm 0.22$ & $\mathrm{bc}$ \\
\hline
\end{tabular}

Values within a column which have no letters in common are significantly different at $\alpha=0.05$ 
Table III. Average abdomen lipid accumulation in newly emerged workers from Pol-line and Russian colonies after 7 days of pollen and spirulina consumption

\begin{tabular}{|c|c|c|c|c|}
\hline \multirow{2}{*}{ Colony } & \multicolumn{3}{|c|}{$\underline{\text { Abdomen lipids }(\mu \mathrm{g} / \mathrm{bee} \pm \mathrm{SE})}$} & \\
\hline & Pollen-fed & & Spirulina-fed & \\
\hline Pol-1 & $515.3 \pm 29.1$ & bcde & $502.5 \pm 46.1$ & $a b c$ \\
\hline Pol-2 & $671.0 \pm 21.6$ & $a b c$ & $571.9 \pm 28.4$ & $\mathrm{ab}$ \\
\hline Pol-3 & $729.9 \pm 16.0$ & $\mathrm{a}$ & $503.8 \pm 44.0$ & $a b c$ \\
\hline Pol-4 & $629.6 \pm 26.5$ & abcde & $653.7 \pm 38.0$ & $\mathrm{a}$ \\
\hline Pol-5 & $640.7 \pm 63.1$ & abcd & $531.0 \pm 41.5$ & $a b c$ \\
\hline Pol-6 & $695.2 \pm 17.2$ & $a b$ & $560.5 \pm 18.9$ & $a b$ \\
\hline Rus-1 & $659.6 \pm 62.8$ & $a b c$ & $548.0 \pm 28.7$ & $a b$ \\
\hline Rus-2 & $449.2 \pm 28.7$ & $\mathrm{e}$ & $330.7 \pm 51.7$ & $\mathrm{c}$ \\
\hline Rus-3 & $613.3 \pm 29.1$ & abcde & $372.4 \pm 48.9$ & $\mathrm{bc}$ \\
\hline Rus-4 & $509.7 \pm 56.6$ & cde & $490.8 \pm 67.0$ & $a b c$ \\
\hline Rus-5 & $537.8 \pm 39.9$ & bcde & $391.6 \pm 49.1$ & $\mathrm{bc}$ \\
\hline Rus-6 & $447.9 \pm 31.6$ & de & $686.9 \pm 13.8$ & $\mathrm{a}$ \\
\hline
\end{tabular}

Values within a column which have no letters in common are significantly different at $\alpha=0.05$

which approximates nurse bee physiology in a colony. While this study is based on experiments with laboratory-reared bees and as such provides some insight into effects on workers, the findings warrant further study on the impacts of genetic variation on nutritional response at the colony level.

Pollen intake was approximately twice as high as spirulina in both stocks. This was consistent with reduced spirulina consumption relative to pollen and pollen substitute diets observed before (Ricigliano and Simone-Finstrom 2020). Ad libitum sugar consumption was highest in bees fed only sucrose and lowest in pollen-fed bees. Honey bees exclusively use aerobic carbohydrate catabolism for energy, which is well-characterized during flight (Beenakkers 1969; Harrison and Roberts 2000), and also during walking and resting (Rothe and Nachtigall 1989). Overall sugar consumption by Russian bees was higher than Pol-line with no differences in feed (protein and lipid) intake. This result may be indicative of an increased basal metabolic rate. Environment and genetics influence flight metabolic rate (Harrison and Fewell 2002), which likely extends to activity and basal metabolism under laboratory conditions. Consistent with our findings, bee genotype influences the average number of daily flights, the total number of foraging flights, and total flight duration, in which Russian honey bees outperformed Italian honey bees (de Guzman et al. 2017).

Table IV. Average vitellogenin (vg) and vg-like-A expression in newly emerged workers from Pol-line and Russian colonies after 7 days of pollen and spirulina consumption

\begin{tabular}{|c|c|c|c|c|c|c|c|c|}
\hline \multirow{3}{*}{$\frac{\text { Colony }}{\text { Pol-1 }}$} & \multicolumn{3}{|c|}{ 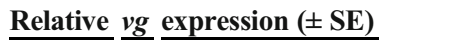 } & & \multicolumn{3}{|c|}{$\underline{\text { Relative }}$ vg-like- $A$ expression ( $\pm \mathrm{SE})$} & \\
\hline & Pollen-fed & & Spirulina-fed & & Pollen-fed & & Spirulina-fed & \\
\hline & $4.73 \pm 0.98$ & $\mathrm{ab}$ & $0.51 \pm 0.18$ & $\mathrm{~b}$ & $0.004 \pm 0.001$ & $\mathrm{bc}$ & $0.009 \pm 0.004$ & $\mathrm{a}$ \\
\hline Pol-2 & $5.36 \pm 1.13$ & $\mathrm{a}$ & $1.45 \pm 0.25$ & $\mathrm{ab}$ & $0.009 \pm 0.003$ & $\mathrm{a}$ & $0.032 \pm 0.011$ & a \\
\hline Pol-3 & $1.91 \pm 0.11$ & $\mathrm{bc}$ & $0.74 \pm 0.33$ & $\mathrm{ab}$ & $0.003 \pm 0.001$ & $\mathrm{c}$ & $0.001 \pm 0.0003$ & a \\
\hline Pol-4 & $2.52 \pm 0.47$ & bc & $1.76 \pm 0.47$ & $\mathrm{ab}$ & $0.006 \pm 0.002$ & $a b c$ & $0.012 \pm 0.005$ & a \\
\hline Pol-5 & $1.28 \pm 0.38$ & $\mathrm{c}$ & $2.51 \pm 0.14$ & $a b$ & $0.011 \pm 0.007$ & $\mathrm{c}$ & $0.001 \pm 0.0004$ & $\mathrm{a}$ \\
\hline Pol-6 & $0.80 \pm 0.09$ & $\mathrm{c}$ & $2.80 \pm 0.59$ & $\mathrm{a}$ & $0.020 \pm 0.010$ & $\mathrm{c}$ & $0.001 \pm 0.0003$ & $\mathrm{a}$ \\
\hline Rus-1 & $1.94 \pm 0.39$ & $\mathrm{bc}$ & $1.84 \pm 0.54$ & $a b$ & $0.015 \pm 0.003$ & $a b$ & $0.031 \pm 0.006$ & $\mathrm{a}$ \\
\hline Rus-2 & $2.78 \pm 0.43$ & $\mathrm{bc}$ & $1.70 \pm 0.36$ & $\mathrm{ab}$ & $0.009 \pm 0.006$ & $a b c$ & $0.021 \pm 0.006$ & $\mathrm{a}$ \\
\hline Rus-3 & $1.76 \pm 0.22$ & $\mathrm{bc}$ & $1.76 \pm 0.48$ & $\mathrm{ab}$ & $0.005 \pm 0.002$ & $\mathrm{c}$ & $0.004 \pm 0.001$ & $\mathrm{a}$ \\
\hline Rus-4 & $1.02 \pm 0.20$ & $\mathrm{c}$ & $1.26 \pm 0.22$ & $\mathrm{ab}$ & $0.011 \pm 0.002$ & $\mathrm{c}$ & $0.005 \pm 0.003$ & a \\
\hline Rus-5 & $1.24 \pm 0.18$ & $\mathrm{c}$ & $2.74 \pm 0.28$ & $\mathrm{a}$ & $0.005 \pm 0.002$ & $\mathrm{c}$ & $0.002 \pm 0.0003$ & $\mathrm{a}$ \\
\hline Rus-6 & $1.21 \pm 0.52$ & $\mathrm{bc}$ & $2.22 \pm 0.79$ & $\mathrm{ab}$ & $0.011 \pm 0.004$ & $\mathrm{c}$ & $0.002 \pm 0.001$ & $\mathrm{a}$ \\
\hline
\end{tabular}


Changes in head and thorax mass represent dietary protein assimilation into brood food-producing hypopharyngeal glands (Crailsheim 1990; DeGrandi-Hoffman et al. 2010) and flight muscles (Hendriksma et al. 2019), respectively. Despite reduced consumption, spirulina-fed bees from both stocks had higher head weights and equal thorax weights compared to pollen-fed bees. This is consistent with high protein bioavailability of spirulina diets determined in commercial Italian bee stock (Ricigliano and SimoneFinstrom 2020). Although newly emerged Pol-line bees had higher head and thorax weights than Russian bees, the stocks experienced equivalent fold increases in weight after consuming pollen and spirulina diets.

The abdominal fat body is the main site of insect nutrient storage and metabolic activities, especially protein synthesis and lipid metabolism ( $\mathrm{Li}$ et al. 2019). In honey bees, the fat body is most developed in nurses, with decreases in lipid and vitellogenin levels shown to precede the transition to foraging (Amdam et al. 2003; Toth and Robinson 2005). Pollen consumption increases fat body lipid stores and polyunsaturated fatty acid (PUFA) abundance, which are positively linked to $v g$ expression (Wegener et al. 2018). Pollen-fed bees had higher lipid and equivalent $v g$ levels relative to spirulina-fed bees. Despite significantly reduced consumption, spirulina led to $88-90 \%$ of the pollen diet-induced lipid content in both stocks. This high bioavailability is consistent with a variety of functional lipids occurring in spirulina (Ricigliano and Simone-Finstrom 2020) including linoleic acid and alphalinoleic acid, two PUFAs that are considered essential for bees (Avni et al. 2014; Arien et al. 2015). Overall, Pol-line accumulated more abdomen lipids and consumed less sugar than Russian bees. Further, Pol-line bees fed pollen had 1.8-fold higher $v g$ levels than pollen-fed Russian bees. These differences may reflect life history-related physiological trade-offs between reproduction and flight capacity. Pol-line is derived from introgression of Varroa resistance into commercial Italian bee stock, which was selected for the favorable management characteristics of colony size and honey production (Delaney et al. 2009). Reproductive potential is generally linked to nutritional stores in social Hymenoptera (Wheeler 1986) and so reduced lipid accumulation in Russian bees is consistent with their colonies tending to have smaller bee populations than Italian colonies (Danka et al. 2006).

The health implications of studying genotype-nutrition interactions has been realized in humans and other model animals over the past decade (Mathers 2017). Nutrigenomics applied to livestock agriculture can be effective in increasing growth rate, productivity, nutrient efficiency, disease resistance, and reproduction (Scholtz et al. 2013; Benitez et al. 2017). Since feed costs comprise a significant expense in animal production, nutrigenomics has potential to bridge the gap between genetic profile and nutrient conversion into augmented productivity. This is best exemplified by the poultry industry, where artificial selection for meatier broiler chickens has facilitated the rapid development of chickens with improved digestive efficiency, favorable body composition, and energy utilization for growth and metabolic activities (Tallentire et al. 2016). One example of how genotype-dependent variation in nutrient efficiency could impact honey bee health is differential capacities to convert available nutrition into vitellogenin or other protein and lipid constituents essential to colony- and individuallevel processes. Furthermore, genetic variation may influence gut microbiome dynamics, which are implicated in nutrient assimilation and feeding behaviors (Ricigliano and Anderson 2020). There are potentially thousands of genetic polymorphisms that may result in variations in nutritional biochemistry influencing honey bee health. While honey bee nutrition is an area of significant research focus, the impact of genetic variation on nutritional response is largely unexplored. Future contrasts between genetic stocks may lead to the identification of metabolic and physiological phenotypes for breeding bees with improved nutrient efficiency and robustness as well as genotype-specific nutritional supplements. 


\section{SUPPLEMENTARY INFORMATION}

The online version contains supplementary material available at https://doi.org/10.1007 /s13592-021-00877-3.

\section{ACKNOWLEDGEMENTS}

We thank members of the USDA-ARS Honey Bee Breeding, Genetics, and Physiology lab for their technical support, and stimulating discussions. We further thank the reviewers for helpful comments and suggestions that improved the manuscript. Mention of trade names or commercial products in this publication is solely for the purpose of providing specific information and does not imply recommendation or endorsement by the US Department of Agriculture is an equal opportunity provider and employer.

\section{AUTHOR CONTRIBUTION}

VAR conceived this research and designed the experiments. VAR, KEI, and STW performed the experiments and analyzed the data. VAR, KEI, and STW wrote the manuscript. All authors read and approved the final manuscript.

\section{FUNDING}

This research was partially funded by the USDAARS research plan 501-2022-050 017 and Agriculture and Food Research Initiative grant no. 2021-67013-33556 from the USDA National Institute of Food and Agriculture.

\section{DATA AVAILABILITY}

The datasets generated during and/or analyzed during the current study are available from the corresponding author on reasonable request

\section{Consent to participate Not applicable}

Consent for publication Not applicable

Competing interests The authors declare no competing interests.

\section{OPEN ACCESS}

This article is licensed under a Creative Commons Attribution 4.0 International License, which permits use, sharing, adaptation, distribution and reproduction in any medium or format, as long as you give appropriate credit to the original author(s) and the source, provide a link to the Creative Commons licence, and indicate if changes were made. The images or other third party material in this article are included in the article's Creative Commons licence, unless indicated otherwise in a credit line to the material. If material is not included in the article's Creative Commons licence and your intended use is not permitted by statutory regulation or exceeds the permitted use, you will need to obtain permission directly from the copyright holder. To view a copy of this licence, visit http://creativecommons. org/licenses/by/4.0/.

Comparaison nutrigénétique de deux souches d'abeilles mellifères résistantes au Varroa nourries au pollen et à la microalgue spirulina.

Apis mellifera / génétique nutritionnelle / élevage / lipide / vitellogénine.

Nutrigenetischer Vergleich zweier Varroa-resistenter Honigbienenstämme, die mit Pollen und SpirulinaMikroalgen gefüttert wurden.

Apis mellifera / Ernährungsgenetik / Zucht / Lipide / Vitellogenin.

\section{CODE AVAILABILITY}

Not applicable

\section{DECLARATIONS}

\section{REFERENCES}

Ethics approval Not applicable

Alaux, C., Dantec, C., Parrinello, H., Le Conte, Y. (2011) Nutrigenomics in honey bees: digital gene expression 
analysis of pollen's nutritive effects on healthy and varroa-parasitized bees. BMC Genomics 12, 496

Amdam, G.V., Norberg, K., Hagen, A., Omholt, S.W. (2003) Social exploitation of vitellogenin, Proc Natl Acad Sci 100, 1799-1802.

Amdam, G.V., Norberg, K., Omholt, S.W., Kryger, P., Lourenço, A.P., Bitondi, M.M.G., Simões, Z.L.P. (2005) Higher vitellogenin concentrations in honey bee workers may be an adaptation to life in temperate climates. Insect Soc 52, 316-319.

Arien, Y., Dag, A., Zarchin, S., Masci, T., Shafir, S. (2015) Omega-3 deficiency impairs honey bee learning. Proc Natl Acad Sci 112, 15761-15766.

Avni, D., Hendriksma, H.P., Dag, A., Uni, Z., Shafir, S. (2014) Nutritional aspects of honey bee-collected pollen and constraints on colony development in the eastern Mediterranean. J Insect Physiol 69, 65-73.

Azzouz-Olden, F., Hunt, A., DeGrandi-Hoffman, G. (2018) Transcriptional response of honey bee (Apis mellifera ) to differential nutritional status and Nosema infection. BMC Genomics 19, 628-20.

Beenakkers, A. T. (1969). Carbohydrate and fat as a fuel for insect flight. A comparative study. J Insect Physiol 15, 353-361.

Benitez, R., Nunez, Y., Óvilo, C., Ovilo, C. (2017) Nutrigenomics in farm animals. J Investig Genomics 4, 1-7.

Brodschneider, R., Crailsheim, K. (2010) Nutrition and health in honey bees. Apidologie 41, 278-294.

Chakrabarti, P., Morré, J.T., Lucas, H.M., Maier, C.S., Sagili, R.R. (2019) The omics approach to bee nutritional landscape. Metabolomics 15, 127-10.

Cheng, Y., Zheng, Y., VanderGheynst, J.S. (2011) Rapid quantitative analysis of lipids using a colorimetric method in a microplate format. Lipids 46, 95-103.

Crailsheim, K. (1990) The protein balance of the honey bee worker. Apidologie 21, 417-429.

Crailsheim, K., Schneider, L. H. W., Hrassnigg, N., Bühlmann, G., Brosch, U., Gmeinbauer, R., \& Schöffmann, B. (1992) Pollen consumption and utilization in worker honeybees (Apis mellifera carnica): dependence on individual age and function. $\mathrm{J}$ Insect Physiol 38, 409-419.

Danka, R.G., Sylvester, H.A., Boykin, D. (2006) Environmental influences on flight activity of USDA-ARS Russian and Italian stocks of honey bees (Hymenoptera: Apidae) during almond pollination. J Econ Entomol 99, 1565-1570.

Danka, R.G., Harris, J.W., Dodds, G.E. (2016) Selection of VSH-derived "Pol-line" honey bees and evaluation of their Varroa-resistance characteristics. Apidologie 47, 483-490.

De Groot, A.P. (1953) Protein and amino acid requirements of the honeybee (Apis mellifica L.). Physiol Comp Oecol 3, 1-83.

de Guzman, L.I., Frake, A.M., Simone-Finstrom, M. (2017) Comparative flight activities and pathogen load of two stocks of honey bees reared in gammairradiated combs. Insects $\mathbf{8}, 127$.

DeGrandi-Hoffman, G., Chen, Y., Huang, E., Huang, M.H. (2010) The effect of diet on protein concentration, hypopharyngeal gland development and virus load in worker honey bees (Apis mellifera L.). J Insect Physiol 56, 1184-1191.

DeGrandi-Hoffman, G., Gage, S. L., Corby-Harris, V., Carroll, M., Chambers, M., Graham, H., deJong, E.W., Hidalgo, G., Calle, S., Azzouz-Olden, F., Meador, C., Ziolkowski, N. (2018). Connecting the nutrient composition of seasonal pollens with changing nutritional needs of honey bee (Apis mellifera L.) colonies. J Insect Physiol 109, 114-124

Delaney, D.A., Meixner, M.D., Schiff, N.M., Sheppard, W.S. (2009) Genetic characterization of commercial honey bee (Hymenoptera: Apidae) populations in the United States by using mitochondrial and microsatellite markers. Ann Entomol Soc Am 102, 666-673.

Ferguson, L.R. (2009) Nutrigenomics approaches to functional foods. J Am Diet Assoc 109, 452-458.

Harbo, J.R., Harris, J. (2005) Suppressed mite reproduction explained by the behaviour of adult bees. J Apic Res 44, 21-23.

Harrison, J.F., Fewell, J.H. (2002) Environmental and genetic influences on flight metabolic rate in the honey bee, Apis mellifera. Comp Biochem Physiol Part A Mol Integr Physiol 133, 323-333.

Harrison, J.F., Roberts, S.P. (2000) Flight respiration and energetics. Annu Rev Physiol 62, 179-205.

Hendriksma, H.P., Pachow, C.D., Nieh, J.C. (2019) Effects of essential amino acid supplementation to promote honey bee gland and muscle development in cages and colonies. J Insect Physiol 117, 103906.

Hölldobler, B., Wilson, E.O. (2009) The superorganism. W. W. Norton \& Company, New York, NY.

Ischebeck, T. (2016) Lipids in pollen - They are different. Biochim Biophys Acta 1861, 1315-1328. https://doi. org/10.1016/j.bbalip.2016.03.023.

Kaspi, R., Mossinson, S., Drezner, T., Kamenzky, B., Yuval, B. (2002) Effects of larval diet on development rates and reproductive maturation of male and female Mediterranean fruit flies. Physiol Entomol 27, 29-38.

Li, S., Yu, X., Feng, Q. (2019) Fat body biology in the last decade. Annu Rev Entomol 64, 315-333.

Lourenço, A.P., Mackert, A., dos Santos Cristino, A., Simões, Z.L.P. (2008) Validation of reference genes for gene expression studies in the honey bee, Apis mellifera, by quantitative real-time RT-PCR. Apidologie 39, 372-385.

Martin, N., Hulbert, A.J., Brenner, G.C., Brown, S.H., Mitchell, T.W., Else, P.L. (2019) Honey bee caste lipidomics in relation to life-history stage and the long life of the queen. J Exp Biol 222. https://doi. org/10.1242/jeb.207043.

Mathers, J. C. (2017) Nutrigenomics in the modern era. Proc Nutr Soc 76, 265-275. 
Mogren, C.L., Margotta, J., Danka, R.G., Healy, K. (2018) Supplemental carbohydrates influence abiotic stress resistance in honey bees. J Apic Res 57, 682-689.

Nabors, R. (2000) The effects of spring feeding pollen substitute to colonies of Apis mellifera. Am Bee J 140, 322-323.

Naug, D. (2009) Nutritional stress due to habitat loss may explain recent honeybee colony collapses. Biol Conserv 142, 2369-2372.

Nestel, D., Papadopoulos, N.T., Pascacio-Villafán, C., Righini, N., Altuzar-Molina, A.R., Aluja, M. (2016) Resource allocation and compensation during development in holometabolous insects. J Insect Physiol 95, 78-88.

Nowacka-Woszuk, J. (2020) Nutrigenomics in livestock-recent advances. J Appl Genet 61, 93-103.

Page, R.E., Fondrk, M.K. (1995) The effects of colonylevel selection on the social organization of honey bee (Apis mellifera L.) colonies: colony-level components of pollen hoarding. Behav Ecol Sociobiol 36, 135144.

Ricigliano, V.A. (2020) Microalgae as a promising and sustainable nutrition source for managed honey bees. Arch Insect Biochem Physiol 104, 1-8.

Ricigliano, V. A., \& Anderson, K. E. (2020) Probing the honey bee diet-microbiota-host axis using pollen restriction and organic acid feeding. Insects 11, 291

Ricigliano, V.A., Simone-Finstrom, M. (2020) Nutritional and prebiotic efficacy of the microalga Arthrospira platensis (spirulina) in honey bees. Apidologie 51, 898-910.

Ricigliano, V.A., Dong, C., Richardson, L.T., Donnarumma, F., Williams, S.T., Solouki, T., Murray, K.K., (2020) Honey bee proteome responses to plant and cyanobacteria (blue-green algae) diets, ACS Food Sci Technol 1.

Rinderer, T.E., Kuznetsov, V.N., Danka, R.G., Delatte, G.T. (1997) An importation of potentially varroaresistant honey bees from far-eastern Russia. Am Bee J 137, 787-789.

Rothe, U., Nachtigall, W. (1989) Flight of the honey bee. J Comp Physiol B 158, 739-749.

Rutter, L., Carrillo-Tripp, J., Bonning, B. C., Cook, D., Toth, A. L., Dolezal, A. G. (2019). Transcriptomic responses to diet quality and viral infection in Apis mellifera. BMC Genomics 20 1-20
Saelao, P., Simone-Finstrom, M., Avalos, A., Bilodeau, L., Danka, R.G., de Guzman, L., Rinkevich, F., Tokarz, P. (2020). Genome-wide patterns of differentiation within and among US commercial honey bee stocks. BMC Genomics 21, 1-12.

Salmela, H., Stark, T., Stucki, D., Fuchs, S. (2016) Ancient duplications have led to functional divergence of vitellogenin-like genes potentially involved in inflammation and oxidative stress in honey bees. Genome Biol Evol 8, 495-506.

Scholtz, M.M., Maiwashe, N., Nesser, F., Theunissen, A., Olivier, W., Mokolobate, M.C., Hendriks, J. (2013) Livestock breeding for sustainability to mitigate global warming, with the emphasis on developing countries. S Afr J Anim Sci 43, 269.

Simopoulos, A.P. (2010) Nutrigenetics/nutrigenomics. Annu Rev Public Health 31, 53-68.

Smart, M., Pettis, J., Rice, N., Browning, Z., Spivak, M. (2016) Linking measures of colony and individual honey bee health to survival among apiaries exposed to varying agricultural land use. PLoS One 11. https://doi.org/10.1371/journal.pone.01526.

Spivak, M., Reuter, G.S. (2001) Varroa destructor infestation in untreated honey bee (Hymenoptera: Apidae) colonies selected for hygienic behavior. J Econ Entomol 94, 326-331.

Tallentire, C.W., Leinonen, I., Kyriazakis, I. (2016) Breeding for efficiency in the broiler chicken: A review. Agron Sustain Dev 36, 66.

Toth, A.L., Robinson, G.E. (2005) Worker nutrition and division of labour in honeybees. Anim Behav 69, 427435.

Trujillo, E., Davis, C., Milner, J. (2006) Nutrigenomics, proteomics, metabolomics, and the practice of dietetics. J Am Diet Assoc 106, 403-413.

Wegener, J., Jakop, U., Schiller, J., Müller, K. (2018) The membrane phospholipid composition of honeybee (Apis mellifera) workers reflects their nutrition, fertility, and vitellogenin stores. Insect Soc 65, 381-391.

Wheeler, D. E. (1986) Developmental and physiological determinants of caste in social Hymenoptera: evolutionary implications. Am Nat 128, 13-34.

Publisher's note Springer Nature remains neutral with regard to jurisdictional claims in published maps and institutional affiliations. 